\title{
Predictive Factors for the Positivity of the Sentinel Lymph Node in Malignant Melanoma
}

\author{
Călin Crăciun, Orsolya Hankó- Bauer, Zalán Benedek, Sorin Sorlea, Marius Florin Coroș, \\ Rareș Georgescu
}

Department of Surgery, University of Medicine and Pharmacy, Tîrgu Mureș, Romania

\section{CORRESPONDENCE \\ Orsolya Hankó- Bauer \\ Str. Gheorghe Marinescu nr. 1 \\ 540103 Tîrgu Mureș, Romania \\ Tel: +40 740698606 \\ E-mail: orsolyabauer@gmail.com}

\section{ARTICLE HISTORY}

Received: September 19, 2017

Accepted: November 25, 2017
Călin Crăciun • Str. Gheorghe Marinescu nr. 1, 540103 Tîrgu Mureș, Romania. Tel: +40 365882588 Zalán Benedek • Str. Gheorghe Marinescu nr. 1 , 540103 Tîrgu Mureș, Romania. Tel: +40 365882588 Sorin Sorlea • Str. Gheorghe Marinescu nr. 1, 540103 Tîrgu Mureș, Romania. Tel: +40 365882588 Marius Florin Coros - Str. Gheorghe Marinescu nr. 1 , 540103 Tîrgu Mureș, Romania. Tel: +40 365882588 Rares Georgescu • Str. Gheorghe Marinescu nr. 1 , 540103 Tîrgu Mures, Romania. Tel: +40 365882588

\begin{abstract}
Malignant melanoma is a neoplasia that has its origin in the melanocytes, the melanin-synthesizing pigment cells present in the epidermis or sometimes in the dermis. Sentinel lymph node biopsy (SLNB) is the standard procedure used for staging patients with malignant melanoma in the majority of surgical centers in the world. With a probability of approximately $20 \%$ of finding positive lymph nodes, it spares a large number of patients of a complete lymphatic dissection. The aim of this study is to evaluate the factors that can predict the positivity of sentinel lymph nodes in malignant melanoma patients. We performed a retrospective study analyzing the histopathologic reports of patients who underwent SLNB for malignant melanoma between 2012 and 2015. There were 32 patients identified, out of which only three (9.37\%) had positive $\mathrm{SLN}$, so the majority of our patients were spared of regional lymphatic dissection. In our series, lymphatic invasion $(p=0.01)$, Breslow index $>4 \mathrm{~mm}(p=0.0064)$, AJCC staging $(p=0.0008)$, the presence of precursory lesions $(p=002)$, and microsatellitosis $(p=0.017)$ were predictive factors for the positivity of the SLN in malignant melanoma patients. Although our results are similar to those published in the literature, we consider that larger cohort studies should be performed to consolidate our results.
\end{abstract}

Keywords: malignant melanoma, sentinel node biopsy, predictive factors

\section{INTRODUCTION}

Malignant melanoma is a neoplasia that has its origin in the melanocytes, the melanin-synthesizing pigment cells present in the epidermis or sometimes in the dermis. ${ }^{1}$ These tumors are usually strongly pigmented, but sometimes they can be amelanotic. They are more aggressive, even the small tumors can develop metastasis; this is what causes the relatively uncertain and unfavorable outcome. ${ }^{1,2}$ Melanomas represent $90 \%$ from the total of deaths associated with cutaneous tumors. ${ }^{3}$

The incidence of malignant melanoma is continuously rising, with a higher rate than other types of cancer, reaching $300 \%$ in the last 40 years. The reason of 
this incidence is unclear, but the more frequent exposure to the sun is speculated, especially at early ages. ${ }^{1,4}$

Sentinel lymph node biopsy (SLNB) is the standard procedure used for staging patients with malignant melanoma in the majority of surgical centers in the world. With a probability of approximately $20 \%$ of finding positive lymph nodes, it spares a large number of patients of a complete lymphatic dissection, a much more invasive procedure, associated with a high mortality and morbidity. ${ }^{5}$

Since in Romania, as well as all over the world, mortality due to malignant melanoma is rising, and the implementation of SLNB can be a benefit in increasing survival and lowering morbidity by reducing the number of complete lymphatic dissections, it is important to analyze the factors that influence the positivity of the sentinel lymph node at a local level.

The aim of this study is to highlight the correlation between the characteristics of the primary tumor that leads to the positivity of the SLN and the impact upon survival.

\section{MATERIAL AND METHODS}

The present study consists of a retrospective analysis of data obtained from patients from the Surgery Clinic of the Mureș County Hospital, who underwent the following procedures between 2012 and 2015, after being diagnosed with malignant melanoma: surgical excision of the tumor and histopathologic analysis, followed by excision of the sentinel lymph node according to the histopathologic result.

The data were extracted from the archive of the Surgery Department; the observation sheets and the histopathological forms were analyzed. The patients were contacted by phone in order to follow the post-surgical evolution and a possible appearance of relapses and metastasis.

\section{The studied group}

The patients were selected for the study based on the inclusion and exclusion criteria listed below.

Inclusion criteria: patients who came to the Surgery Department of the Tîrgu Mureş County Hospital with the diagnosis of malignant melanoma, confirmed through a histopathologic examination; patients who have undergone sentinel lymph node biopsies between January 1, 2012 and December 31, 2015.

Exclusion criteria: patients investigated or diagnosed in other departments or clinics; patients who have been investigated at the Surgery Department of the Tîrgu Mureş County Hospital at another time than between January 1,
2012 and December 31, 2015; patients with insufficient data; patients in whom no sentinel lymph node was found.

Being a relatively recently implemented technique at a local level, as a result of applying the above-mentioned criteria, we have selected an initial group of 35 patients, from which three were eliminated due to the lack of data, and two patients were eliminated because no sentinel lymph node was not found.

Before the surgical procedure, the patient undergoes a physical examination in order to identify or rule out the presence of additional primary melanoma, in transit or satellite, and any sign of lymphatic adenopathy. Radiography, computed tomography (CT) or positron-emission tomography (PET) are not recommended in the absence of clinical suspicion, since the metastatic lesions at a distance, which are detectable by radiography, do not show in the primary stages of melanoma in asymptomatic patients.

In patients where there is a clinical suspicion of ganglionic metastasis, biopsy of the sentinel lymph node is justified. In these cases, ultrasound examination along with biopsy with a thin needle must be taken into consideration. A negative result at the aspiration with a thin needle does not rule out the absence of ganglion metastasis, and SLN biopsy still remains indicated.

During SLN biopsy, a radio-marked colloid (Tc 99) is injected around the area of the primitive tumor. Then a device is used to locate the radioactive lymph nodes (considered sentinel lymph nodes), after which they are excised and sent to histopathologic examination.

Following the excision of the marked sentinel lymph nodes, a large local excision of the primary tumor is done, in order to reduce the possibility of a local relapse due to microsatellitosis. The rescanning of the relevant lymphatic groups is made in order to identify the on route (interval) or ectopic sentinel ganglions with the help of the gamma sample, to ensure the excision of all SLN. For patients with negative SLN, other interventions on the lymph node group are not recommended, although in a stage IIB patient adjuvant therapy would be recommended; for patients with at least one positive SLN, complete regional lymph node dissection is the standard treatment, following a discussion with the patient about the risk of having other ganglionic metastases.

The following variables were used in the study: gender and age; location of the primary tumor and the lymphatic drainage area (the area where the sentinel lymph node was found); the number of excised lymph nodes and the number of the positive lymph nodes; the dimensions of the tumor and of the sentinel node; the Breslow index and the Clark level; the TNM and AJCC stages; the growth on the 
radial or on the vertical axe; the presence or the absence of ulceration; the histologic type (nodular or lentiginous); the presence of vascular, lymphatic, or perineural invasion; regression; the presence of intratumoral lymphatic inflammatory infiltrate; desmoplasia; microsatellitosis; associated or precancerous lesions; complete lymphatic dissection; response to therapy or relapse; reoperations associated with the pathology; and the associated diseases.

Statistical analysis was performed using Microsoft Excel 2010 and GraphPad Prism 6 (Fisher test and Chi square test). A value of $p<0.05$ was considered statistically significant.

\section{RESULTS}

We included in the study a number of 32 cases, out of which three had positive sentinel lymph node at the histopathologic examination. The studied group was made up of 14 men and 18 women. The average age of the studied population was 57 years, the youngest age at which the SLN biopsy was made being 33 years, and the oldest 72 years. There was no statistically significant relation between the gender of the patients and the positivity of the sentinel lymph node $(\mathrm{p}=0.4)$.

Localization: The tumors were located in $40.62 \%$ at the abdominal level, $28.12 \%$ at the level of the upper limb, $25 \%$ at the level of the lower limb, and the smallest incidence was found at the level of the head and throat (6.25\%). There was no statistically significant relation between the location of the tumors and the positivity of the sentinel lymph node $(\mathrm{p}=0.27)$.

The characteristics of the primary tumor: The average dimension of the primary tumor was $10 \mathrm{~mm}$ (range 5-35 $\mathrm{mm}$ ). The relation between the size of the tumor and the positivity of the sentinel lymph node was not statistically significant $(\mathrm{p}=0.66)$. From the analyzed tumors, $56.25 \%$ were lentiginous melanomas (17 cases), from which only one had metastasis in the sentinel lymph node. Nodular melanoma was present in $43.75 \%$ of cases ( 12 cases), from which two presented a positive sentinel lymph node at the histopathologic examination. The statistical analysis did not reveal a significant correlation between the histologic types of the primary tumors and the positivity of the sentinel lymph node $(\mathrm{p}=0.35)$.

In our series, 22 cases presented intratumoral lymphatic inflammatory infiltrate, out of which three cases had positive sentinel lymph node biopsy. Although this relation is not significant statistically $(\mathrm{p}=0.54)$, the odds ratio $(\mathrm{OR}=$ 3.769) suggests that there is a high chance to have positive SLN if the primary tumor contains inflammatory infiltrate.
Of all cases, $84.38 \%$ presented a radial increase, and $59.38 \%$ showed increase on the vertical axe, some melanomas showing both radial and vertical increase. Both for the radial and vertical tumor development we did not find a statistically significant relation between the form of growth and SLN positivity ( $\mathrm{p}=0.37$ and $\mathrm{p}=0.77$, respectively). We noticed that 2 out of 3 positive SLN cases presented at the biopsy an ulceration of the primary tumor. The association between the presence or the absence of the tumoral ulceration, and the positivity of the sentinel lymph node is not significant ( $\mathrm{p}=0.21$ ).

Five cases (15.63\%) presented vascular invasion, out of which only one case had metastatic sentinel lymph node. We found no statistically significant relation between the presence of vascular invasion and the positivity of the sentinel ganglion $(\mathrm{p}=0.37)$. From the three $(9.38 \%)$ cases that showed lymphatic invasion, two had metastasis in the sentinel lymph node, which means that there is a statistically significant relation between the presence of lymphatic invasion and the positivity of the sentinel lymph node $(\mathrm{p}=0.01$, Figure 1$)$.

The Breslow index and the Clark level: Most of the primary tumors (53.12\%) had a Breslow index between 1 and $2 \mathrm{~mm}$, while lesions with a thickness between 2 and $4 \mathrm{~mm}$ were found in $15.62 \%$ of the cases, and those with a Breslow index $>4 \mathrm{~mm}$ in $18.75 \%$ of cases. The average Breslow index was $2.11 \mathrm{~mm}$. The Breslow index represents a predictive factor in SLN positivity; the bigger the thickness of the tumor, the higher the chances of a lymphatic metastasis development: all three cases with positive sentinel lymph node had the Breslow index higher than $4 \mathrm{~mm}$, so in our series there was a statistically significant correlation between the Breslow index and the positivity of the sentinel lymph node ( $\mathrm{p}=0.064$, Figure 2$)$.

Even if two out of 10 cases that showed a Clark III level had a positive SLN at the biopsy, and one out of two with a Clark V level showed ganglion metastasis ( $p=0.12$, nonsignificant), the increase of the Clark level did not turn out to be a predictive factor.

Staging: Two out of the three cases who had positive SLNB were in stage T4 according to the TNM staging system, and one case was in stage T1. In our series, the TNM stage of the primary tumor did not influence the positivity of the sentinel lymph node $(\mathrm{p}=0.38)$. Staging according to the AJCC seems to be much more reliable as a predictive factor for the positivity of the SLN. The case that had positive SLN was in stages IIIA, IIIB, and IIIC ( $\mathrm{p}<0.0008$, Figure 3 ).

Precursory lesions: Besides the main tumor, in $12.50 \%$ of the cases we also found associated lesions (melanoma in situ), which might be associated with a worse progno- 


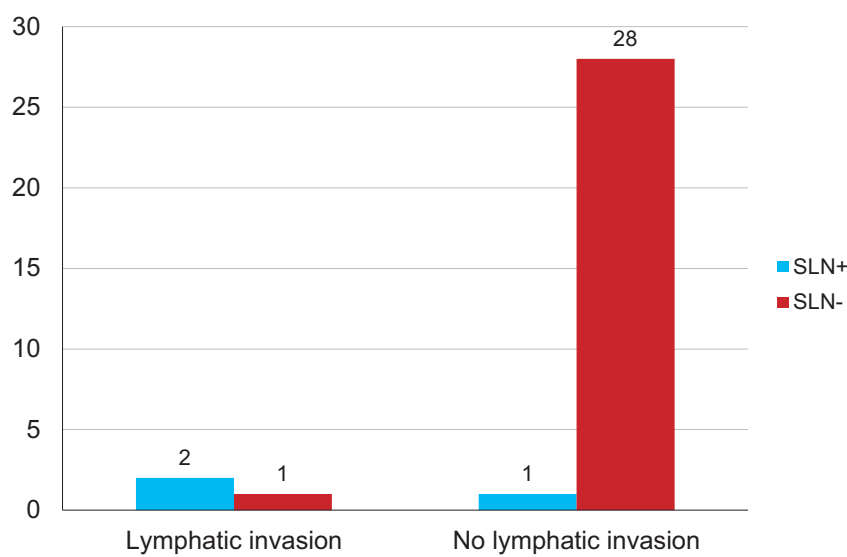

FIGURE 1. Correlation between the presence of lymphatic invasion and the positivity of the sentinel lymph node (SLN: sentinel lymph node)

sis. A total of $15.62 \%$ from the tumors started from precursory lesions: there was a case of melanocytic nevi, one of intradermic nevi, and three cases of junctional nevi, one of them in the context of Hutchinson melanosis. All cases that presented positive SLN started from precursory lesions. In our series, the presence of precursory lesions had a statistically significant influence on the positivity of the sentinel lymph node ( $p<0.002$, Figure 4 ), but the association with melanoma in situ did not influence the metastasis into the sentinel lymph node ( $\mathrm{p}=0.25)$.

Lymphatic drainage: The most frequent area of lymphatic drainage was the axillary region with a percentage of $62.50 \%$; the inguinal area was the drainage headquarters in $28.13 \%$ of the cases, followed by the cervical area in only $9.38 \%$ of the cases. In our case study, the positivity of the sentinel lymph node was not influenced by the area of lymphatic drainage $(\mathrm{p}=0.23)$.

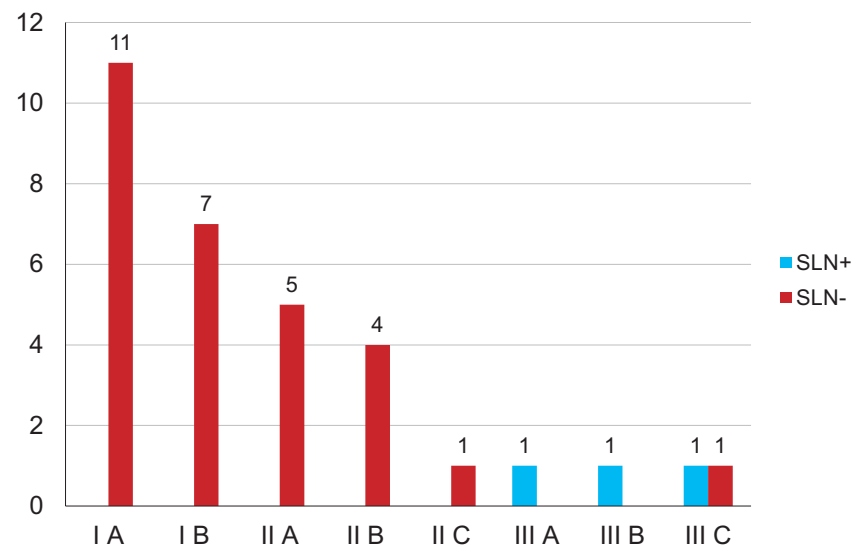

FIGURE 3. Correlation between the AJCC staging of the primary tumor and the positivity of the sentinel lymph node

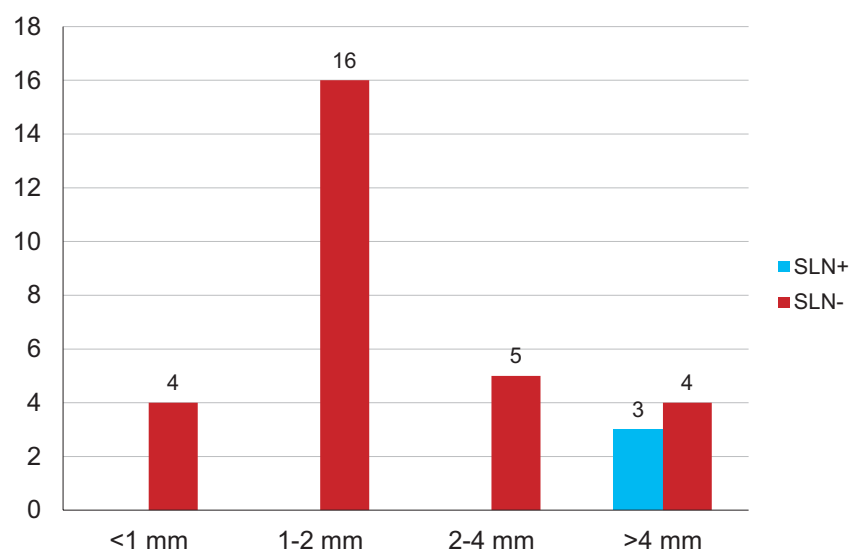

FIGURE 2. Correlation between the Breslow index of the primary tumor and the positivity of the sentinel lymph node (SLN: sentinel lymph node)

Excised lymph nodes: An average of 2.3 lymph nodes were excised (range 1-4). The average diameter of the SLN was of $16.23 \mathrm{~mm}$, their dimensions varying from 3 to $48 \mathrm{~mm}$. Of the total number of excised lymph nodes, only $9.37 \%$ were positive at the histopathologic examination; in these cases complete regional lymph node dissection was performed.

The presence of desmoplasia did not seem to be a predictive factor. Only one patient from the three who showed desmoplasia also had positive SLN at the biopsy ( $p=0.13$ ).

The presence of microsatellitosis was a predictive factor according to the casuistry, one out of two cases who presented microsatellitosis also had ganglion metastasis ( $p=0.017$, Figure 5).

Post-treatment, the outcome was favorable in $75 \%$ and unfavorable in $25 \%$ of the cases, out of which $12.5 \%$ died, and the other half developed metastasis: one case devel-

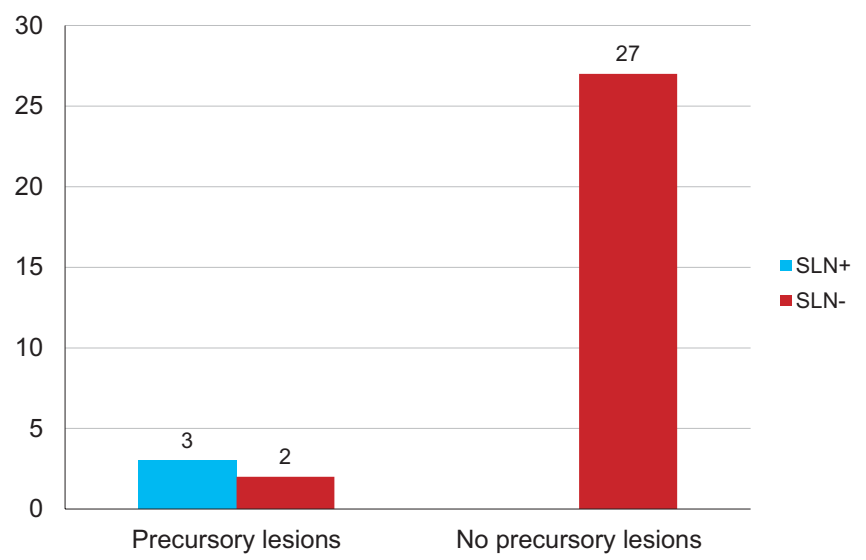

FIGURE 4. Correlation between the presence of precursory lesions and the positivity of the sentinel lymph node 


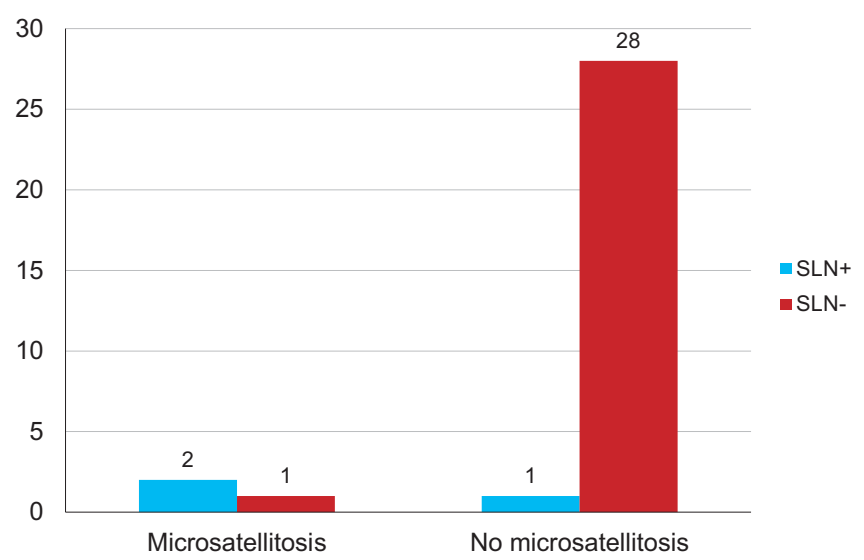

FIGURE 5. Correlation between microsatellotosis and the positivity of the sentinel lymph node

oped pulmonary and cerebral metastasis, two had only their lung affected, one relapsed locally, and the last one developed a tegumentary metastasis at the breast and liver level. Twenty-five per cent of the patients developed granuloma of post-surgical foreign body, while $12.5 \%$ of the patients also had excision interventions on other nevi. There was no correlation between SLN positivity and post-therapy response. There was no significant association between SLN positivity and the forming of foreign body granuloma or the intervention on other nevi.

\section{DISCUSSIONS}

The results of this study suggest that the Breslow index, lymphatic invasion, location, AJCC standardization (which comprises the thickness/Clark level and the ulceration), the presence of precursory lesions, microsatellitosis, and the absence of intratumoral lymphatic inflammatory infiltrate are predictive factors for the positivity of the sentinel lymph node.

These data are comparable with those of other studies: Tejera-Vaquerizo et al., in a study carried out on 818 patients between 2000 and 2012 and published in 2015, found a statistically significant association between the Breslow index, the presence of ulceration, the absence of regression, the low mitotic rate $\left(\geq 6 / \mathrm{mm}^{2}\right)$, and the positivity of the sentinel lymph node, all these representing independent factors. The most important predictive factor was the Breslow thickness. The absence of inflammatory infiltrate, the patient's age, the location of the primary tumor, and microsatellitosis were predictive factors for ganglion metastasis in patients with tumors thicker than 2 $\mathrm{mm}$, these factors together with SLN+ being also predictive for survival. ${ }^{2}$
In another study published by Kibrité et al., including 957 patients ( $52 \%$ men) with an average age of 57 years, the average Breslow index was $1.5 \mathrm{~mm}$ and the most frequent location of the tumors was at the level of the limbs. SLN was found positive in $21.3 \%$ of the patients. The risk factors identified for SLN positivity were the Breslow index, the presence of ulceration, microsatellitosis, lymphovascular invasion, but also the location of the tumor. ${ }^{6}$

In an analysis of a group of 221 patients by Ellis et al., on which sentinel lymph node biopsy was successfully done between 2004 and 2010, SLN was found positive in 48 patients (21.7\%). Only two out of 105 patients with a T1 lesion had positive SLN. It was discovered that male gender, the type of the tumor, the Breslow index (over $2 \mathrm{~mm}$ ), and the absence of intratumoral lymphatic infiltrate were significantly associated with the positivity of the SLN, the last two being independent predictive factors. Location in the upper extremity and the presence of ulceration are additional predictive factors. Most patients who had positive lymph node at the biopsy followed complete lymphatic dissection. The survival at a five-year interval was $53.1 \%$ for SLN+ patients and $88.2 \%$ for SLN- patients. The Breslow index and the SLN status were independent predictive factors for global survival. ${ }^{4}$

There are controversies regarding the purpose of SLN biopsy in patients with tumors thicker than $4 \mathrm{~mm}$, as they present a higher risk of developing metastasis, even after treating the primary illness. Whatever the case, this procedure remains the most important independent factor of survival, even in these patients, according to Morton. ${ }^{7}$

Patients with lesions below $0.75 \mathrm{~mm}$ seem to have an excellent prognosis and are not candidates for a ganglion dissection; at the other extreme, patients with primary tumors with a Breslow index over $3.50 \mathrm{~mm}$ present a very high risk of metastasis at a distance; thus, the benefit of an elective ganglion dissection is undeniable. According to Longo et al., patients with a stage II AJCC melanoma of intermediary sizes could benefit from an elective dissection of the regional lymph nodes for a better prognosis, but there is no common agreement that these patients should undergo such a procedure. ${ }^{1}$

The management of lymph nodes is controversial, as some studies recommend elective ganglion dissection for all patients with melanoma, regardless of the clinical evidence of regional metastatic effect. Anyway, the routine dissection exposes the patients to complications and does not bring a benefit to the majority, those without nodular involvement. $^{8}$

Since there was dissatisfaction with elective ganglion dissection, with the discovery of the sentinel ganglion, lymphatic mapping and SLN biopsy were introduced for 
the individualized management of regional lymph nodes. ${ }^{9}$

Research was carried out in order to see if patients with melanoma who have negative SLN and do not show clinical signs of other nodular involvement can be spared of other extensive surgical procedures at the moment of the primary tumor excision. A meta-analysis of 71 studies which gathered data from 25,240 patients answers affirmatively. According to this, the risk of regional nodular recurrence in patients with negative SLNB is lower than 5\%. ${ }^{4}$

Another question asked by the researchers is whether ganglion dissection following a positive SLN biopsy has a therapeutic benefit for patients regarding the disease-free survival and the survival with melanoma. In order to answer this question, the National Cancer Institute, the National Institute of Health, and the John Wayne Cancer Institute are sponsoring a study called Multicenter Selective Lymphadenectomy Trial II (MSLT-II), where more than 1,900 patients with positive SLN, but without clinical records of other nodular involvement will be treated randomly either by complete lymph node dissection (CLND) or by ultrasonography following the remaining nodules and CLND if there are signs of additional lymphatic metastasis. Although the answer is long waited for, the study is still in progress.

Until then, the MSLT-I study showed the value of SLN biopsy as an independent and unqualified prognostic factor and also a means of efficient control of the illness with the lowest morbidity and mortality associated. ${ }^{10}$

\section{Study limitations}

Due to the relatively short interval of time and to the fact that we are not a specialized center in this pathology, we analyzed a small number of cases. We consider that extending on a longer term or carrying out a multi-centric study would be very useful to consolidate the information we have found.

\section{CONCLUSIONS}

Sentinel lymph node biopsy has become a useful instrument both in the standardization of malign melanoma without any clinically apparent impairment and in the treatment of ganglion metastasis without associating the invasion and the morbidity related closely to complete lymphadenectomy, while also being a prognostic factor.

In our study, most of the patients had no metastasis in the sentinel lymph node, which means that owing to sentinel lymph node biopsy, most patients were exempted from a useless surgical intervention (complete ganglion dissection), with an important morbidity.

The factors that can predict the positivity of the sentinel ganglion are the Breslow index $>4 \mathrm{~mm}$, the AJCC stage of the primary tumor, the presence of precursory lesions, the presence of lymphatic invasion, and microsatellitosis.

\section{CONFLICT OF INTEREST}

Nothing to declare.

\section{REFERENCES}

1. Longo DL, Kasper DL, Jameson JL, et al. Harrison's Principles of Internal Medicine, 18th edition, The McGraw-Hill Companies/United States of America; 2012. p. 723-730.

2. Tejera-Vaquerizo A, Martín-Cuevas P, Gallego E, et al. Predictors of Sentinel Lymph Node Status in Cutaneous Melanoma: A Classification and Regression Tree Analysis. Actas Dermosifiliogr. 2015;106:208-218.

3. Garbe C, Peris K, Hauschild A, et al. Diagnosis and treatment of melanoma: European consensus-based interdisciplinary guideline. Eur J Cancer. 2010;4:270-283.

4. Ellis MC, Weerasinghe R, Corless $\mathrm{CL}$, et al. Sentinel lymph node staging of cutaneous melanoma: predictors and outcomes. Am J Surg. 2010;199:663668.

5. Teixeira V, Vieira R, Coutinho I, et al. Prediction of Sentinel Node Status and Clinical Outcome in a Melanoma Centre. Journal of Skin Cancer. 2013;2013:7.

6. Kibrité $\mathrm{A}$, Milot $\mathrm{H}$, Douville $\mathrm{P}$, et al. Predictive factors for sentinel lymph nodes and non-sentinel lymph nodes metastatic involvement: a database study of 1,041 melanoma patients. Am J Surg. 2016;211:89-94.

7. Morton ML, Thompson JF, Cochran AC, et al. Final Trial Report of SentinelNode Biopsy versus Nodal Observation in Melanoma. $N$ Engl J Med. 2014;370:599-609.

8. Gershenwald JE, Ross MI. Sentinel-Lymph-Node Biopsy for Cutaneous Melanoma. N Engl J Med. 2011;364:1738-1745.

9. Choi EA, Gershenwald JE. Imaging studies in patients with melanoma. Surg Oncol Clin N Am. 2007;16:403-430.

10. Faries MB, Cochran AJ, Elashoff RM, et al. Multicenter Selective Lymphadenectomy Trial-I confirms the central role of sentinel node biopsy in contemporary melanoma management. $\mathrm{Br} J$ Dermatol. 2015;172:571-573. 